\title{
Acute Pancreatitis Induced by Methimazole Therapy
}

\author{
Albin Abraham $^{\mathrm{a}} \quad$ Pooja Raghavan $^{\mathrm{b}} \quad$ Rajshree Patel $^{\mathrm{a}}$ \\ Dhyan Rajan $^{\text {a }}$ Jaspreet Singh $^{a}$ Paul Mustacchia ${ }^{a}$ \\ ${ }^{a}$ Department of Internal Medicine, Nassau University Medical Center, \\ East Meadow, N.Y., and ${ }^{\mathrm{b}}$ Department of Internal Medicine, Mount Carmel \\ Health, Columbus, Ohio, USA
}

\section{Key Words}

Methimazole $\cdot$ Adverse drug reactions · Drug-induced pancreatitis

\begin{abstract}
Among the causative factors for acute pancreatitis, adverse drug reactions are considered to be rare. The diagnosis of drug-induced pancreatitis (DIP) is challenging to establish, and is often underestimated because of the difficulties in determining the causative agent and the need for a retrospective re-evaluation of the suspected agent. We present the case of an 80-year-old woman who presented with complaints of abdominal pain. Her medications included methimazole (MMI) which she had been on for the past 3 months. Computed tomography of her abdomen showed peripancreatic fat stranding with trace amount of surrounding fluid, along with amylase and lipase levels suggestive of acute pancreatitis. In the absence of classical risk factors for acute pancreatitis, a diagnosis of DIP secondary to $\mathrm{MMI}$ use was made. Withdrawal of the drug from her medication regimen was accompanied by relief of symptoms and resolution of clinical evidence of pancreatitis. The aim of this paper is to report only the fourth case of MMI-induced pancreatitis in the published literature, and to illustrate the significance of an appropriate and timely diagnosis of DIP.
\end{abstract}

\section{Introduction}

The incidence of acute pancreatitis is increasing worldwide, and it now ranks as the second most common inpatient gastrointestinal diagnosis in the United States. The economic burden of acute pancreatitis is tremendous, with an estimated 2.5 billion dollars spent annually on inpatient and outpatient medical services directed towards the disorder. In 2000 alone, an estimated 2,834 deaths in the United States occurred from acute pancreatitis, making it the 14th most common cause of death due to gastrointestinal disease [1]. While alcohol and gallstones account for the most 
important etiologic factors for acute pancreatitis, the impact of other agents such as prescribed drugs is being increasingly appreciated.

The incidence of drug-induced pancreatitis (DIP) is generally estimated from case reports or as a result of studies designed and conducted for other purposes. DIP has a clinical manifestation similar to acute pancreatitis from other causes, but the diagnosis is difficult to establish, mainly stemming from the absence of cause-specific diagnostic tests. The diagnosis of DIP usually hinges on four criteria: (1) acute pancreatitis occurs during the administration of the drug; (2) all other common causes of acute pancreatitis are excluded; (3) symptoms of acute pancreatitis disappear after drug withdrawal; (4) symptoms recur after re-challenge of the suspected drug [2].

Methimazole (MMI), which belongs to the imidazole class of antithyroid medications, has long been the mainstay of therapy for patients with Grave's disease and other hyperthyroid states. Although known to be one of the most reliable methods of achieving the euthyroid state, the medication does not come without a myriad of adverse effects. The most common side effects reported from use of MMI include fever, pruritus, and nausea. Less frequently encountered adverse effects such as granulocytopenia, drug-induced lupus, and severe arthralgia should be suspected in patients with such abnormities while on MMI. DIP from MMI use is rare, with only three known cases reported to date, although the true incidence of MMI-induced acute pancreatitis is unclear [3-5].

The true incidence of DIP remains largely unknown. There has been considerable underreporting of DIP in particular due to the fact that the index of suspicion for DIP is lower compared with drug-induced liver disease and other potential medication side effects. Also, milder cases evident by significant but not critical elevations of serum amylase and lipase are often missed by clinicians, unless heightened suspicion of DIP is present. It often takes an astute physician to recognize DIP, given the differences in latent period between the time of drug exposure and the time of development of acute pancreatitis. Unfortunately, many cases of DIP are erroneously classified as due to alcohol or gallstones. Treatment often includes making the patient nil per os and thus inadvertently discontinuing a possible offending medication and missing the opportunity to diagnose DIP. Finally, the true incidence of DIP remains largely unknown because often when a case of DIP is correctly identified, it is seldom reported $[6,7]$.

Here we present the case of a patient who presented with abdominal pain and was diagnosed with MMI-induced pancreatitis.

\section{Case Report}

An 80-year-old Caucasian woman presented to the emergency room with complaints of epigastric pain. She described the pain as being sharp, continuous for the past 2 months, progressively increasing in intensity, worsened with food intake and associated with nausea. She denied any fever, vomiting, diarrhea, constipation, blood per rectum, weight loss, sick contacts or any recent travel. She also denied any yellowing of the eyes, joint pains or rashes on her skin. Her past medical history was significant for hypertension and hyperthyroidism which had been diagnosed 3 months prior. Her home medications included aspirin $81 \mathrm{mg}$ and MMI $10 \mathrm{mg}$. She did not have a history of drug allergies. She denied the use of tobacco, alcohol, illicit drugs, and the use of any supplemental tablets. Family history was unremarkable including the absence of any gastrointestinal disorders. 
On physical examination, the patient appeared mildly anxious but in no apparent distress. Her vital signs were stable. Examination of her abdomen revealed mild epigastric tenderness without rebound tenderness, guarding or rigidity. There was neither abdominal distension nor organomegaly, and bowel sounds were noted to be normal. A complete blood count and basic metabolic profile were within normal limits. Liver-related tests revealed alanine aminotransferase of $40 \mathrm{IU} / \mathrm{l}$, aspartate aminotransferase of $55 \mathrm{IU} / \mathrm{l}$ and alkaline phosphatase of $133 \mathrm{IU} / \mathrm{l}$. Also remarkable was a serum amylase level of $371 \mathrm{IU} / \mathrm{l}$, a lipase level of $581 \mathrm{IU} / \mathrm{l}$ and a lactate dehydrogenase level of $251 \mathrm{IU} / \mathrm{l}$. Cardiac enzymes and a lipid panel were within normal limits. An abdominal ultrasound was performed which showed no evidence of cholelithiasis. Computed tomography of her abdomen revealed peripancreatic fat stranding with trace amount of surrounding fluid suggestive of acute pancreatitis (fig. 1). There was no evidence of biliary duct dilatation. A provisional diagnosis of DIP due to MMI use was made in the absence of other risk factors for acute pancreatitis. She was started on intravenous fluids and given supportive therapy. MMI was discontinued from her drug regimen. After 4 days of conservative management, the patient showed clinical improvement with resolution of her abdominal pain and normalization of lipase levels. She was later discharged from the hospital and has been doing well since. The patient refused a re-challenge with MMI.

\section{Discussion}

Drugs are seldom considered to cause acute pancreatitis, although the World Health Organization (WHO) database listed 2,479 episodes suspected of being caused by 525 different drugs between 1968 and 1993 [8]. It is difficult to determine the true incidence of DIP due to the lack of consistent reporting and lack of large prospective trials. Most information has been communicated in the form of single case reports either reported to local drug safety committees or published as case reports or letters to the editor in peer-reviewed journals. Summaries of the available data suggest that between 0.1 and $2 \%$ of cases of acute pancreatitis may be drug-induced, with a retrospective case study from the Czech Republic finding the incidence rate to be as high as $5.3 \%[2,9]$.

Epidemiological data suggest that certain populations may be at higher risk for DIP, including pediatric patients, the elderly, patients with advanced human immunodeficiency virus (HIV) infection, patients with inflammatory bowel disease, and patients prescribed polypharmacy. The increased risk in children may be conferred as traditional risk factors for pancreatitis such as alcohol abuse are rarely present. In the elderly, polypharmacotherapy and resulting drug interactions may increase the risk of DIP. In patients with advanced HIV infection, the incidence of DIP has been reported to be as high as $14 \%$. Patients with advanced HIV infection may be more susceptible to DIP due to various factors, including impaired balance of CD4 and CD8 cells, increased rate of exogenous and nosocomial infections, and polypharmacy with antiretroviral medications. Those with inflammatory bowel disease are believed to suffer from an increased risk for DIP due in part to their underlying disease, rather than medications alone. For example, acute pancreatitis as an adverse reaction to azathioprine is rarely noted if used for the treatment of conditions other than inflammatory bowel disease, such as rheumatoid arthritis, systemic lupus erythematosus or after kidney transplantation. This supports the hypothesis that inflammatory bowel disease in itself bears an increased risk for acute pancreatitis that is independent of the medication used for therapy, or that patients with inflammatory bowel disease have a predilection for azathioprine-induced pancreatitis. The effect of polypharmacy and combination drug therapy on the increased incidence of acute pancreatitis was highlighted in a case-control study from Denmark. One example given in that study described that the 
risk of developing acute pancreatitis from metronidazole was increased 8-fold if patients received metronidazole in combination with other drugs used to eradicate Helicobacter pylori, such as proton pump inhibitors and amoxicillin $[9,10]$.

The pathophysiologic mechanism of DIP is not well established for many drugs including MMI; however the literature suggests that a few medications do indeed have a distinct mechanism for causing DIP. A direct toxic effect to the pancreas may explain the sequence of events in drugs like metronidazole, which is a medication known to diffuse into the pancreas. In other drugs such as tetracycline, the pathophysiology may involve accumulation of toxic metabolites in the pancreas, resulting in acute inflammation. The putative mechanisms by which codeine causes acute pancreatitis have been postulated to be due to sphincter of Oddi spasm, resulting in eventual pancreatic inflammation. The effect of estrogen supplementation on the pancreas may be indirect with alterations in the plasma triglyceride level possibly contributing to development of acute pancreatitis. In the case of chlorothiazide-induced acute pancreatitis, the potential mechanisms include thiazide-induced hyperparathyroidism, hyperlipidemia, hypercalcemia or hypotension, resulting in pancreatic ischemia. Angiotensin-converting enzyme inhibitors (ACEIs) are well documented to potentially result in DIP, as angioedema of the pancreatic duct due to an interaction with the kallikrein-kinin system could explain some cases. Another theory suggests that ACEIs disturb the pancreatic microcirculation and ductal anion secretion by changing the physiology of the pancreatic renin-angiotensin system. Overdose of medications not classically associated with DIP has also been reported, including paracetamol, erythromycin, carbamazepine and codeine $[2,9,11]$.

The diagnosis of DIP can be challenging for clinicians. If evidence of acute pancreatitis has been established, common etiologies such as gallstones and alcohol abuse need to be excluded. A careful review of all medications taken by the patient should be recorded, and if the patient takes any drug suspected to cause pancreatitis, this should be discontinued if safely possible. If it is not possible to stop the medication, it should be substituted for an alternative medication, if possible from another class of substances. If symptoms stop after the drug has been discontinued, a diagnosis of DIP is probable. Re-exposure to the medication should only be considered if the potential benefits outweigh the risks. Fig. 2 summarizes the approach to a case of DIP [6].

The most extensive overview of DIP was published by Trivedi et al. [6], in which all reported cases of DIP via the National Library of Medicine/PubMed from 1966 to 2004 were reviewed. In this review, medications implicated in cases of DIP were classified based on incidence and medication re-challenge data. Class I medications were defined as those implicated in greater than 20 cases of acute pancreatitis, with at least one documented case following re-exposure. Class II medications were involved in more than 10 but fewer than 20 cases, with or without a positive re-challenge. Class III medications included all other drugs reported to be associated with DIP. Table 1 lists the drugs reported to be class I medications according to the classification method used by Trivedi et al. [6]. As there are less than 10 cases of MMI-induced pancreatitis reported, this drug would be considered as class III in regards to the relative risk for DIP.

Karch and Lasagna [12] classified drugs based on evidence for adverse drug reactions into definite, probable and possible causes. According to this classification, 
the causality was definite if a drug reaction followed the administration of the drug in a temporal sequence and in a known response pattern. Moreover this should be confirmed by cessation of the drug (de-challenge) with reappearance of symptoms upon repeated exposure to the drug (re-challenge). A drug reaction was probable if the reaction followed administration of the drug in a reasonable temporal sequence with symptoms abating on de-challenge. Re-challenge was never attempted but the symptoms could not be explained by the known characteristics of the patient's clinical state. Possible cause, the weakest causality defined, was if the drug reaction followed a temporal sequence from administration of the drug, but the symptoms could also be explained by the patient's clinical state or other modes of therapy administered to the patient.

Vinklerova et al. [2], Andersen et al. [7], Barreto et al. [11], Lankisch et al. [13] and Eland et al. [14] suggest that the incidence of DIP ranges from 0.1 to $5.3 \%$. In these studies, 10 different drugs were found to have a definite causality with acute pancreatitis ( table 2), with the time relationship between intake of the drug and onset of acute pancreatitis varying from 1 day to 17 years. Among the drugs with a definite causality of DIP, death was reported only with valproate and azathioprine. Some of the drugs found to have a probable or possible causality to acute pancreatitis included alendronate, allopurinol, doxycycline, famotidine, hydrochlorothiazide, ibuprofen, lithium, ramipril and rifampin.

In conclusion, it is possible to state that DIP is not a negligible disease. The diagnosis seems to be underestimated because of the difficulties in determining the causative agent and need for a retrospective re-evaluation of the suspected causative factors. This was highlighted by Spanier et al. [15] who in a recent multicenter observational study reported that in $4.8 \%$ of patients a diagnosis of DIP was missed, possibly leading to the patient's detriment. Despite the moderate severity of DIP, all patients with acute pancreatitis of unknown etiology should be carefully questioned regarding current medication use. Clinicians should be cognizant of the rare but relevant incidence of MMI-induced pancreatitis, allowing for timely diagnosis and management of DIP. Once identified, offending agents should be discontinued to reduce further episodes of acute pancreatitis.

\section{Disclosure Statement}

The authors have no funding or conflicts of interest to disclose. 
Table 1. Class I drugs showing the number of cases following re-exposure as reported by Trivedi et al. [6]

\begin{tabular}{llc}
\hline Drugs & $\begin{array}{l}\text { Reported } \\
\text { cases }\end{array}$ & $\begin{array}{l}\text { Cases following } \\
\text { re-exposure }\end{array}$ \\
\hline Didanosine & 883 & 9 \\
Asparaginase & 177 & 2 \\
Azathioprine & 86 & 16 \\
Valproic acid & 80 & 11 \\
Pentavalent antimonials & 80 & 14 \\
Pentamidine & 79 & 2 \\
Mercaptopurine & 69 & 10 \\
Mesalamine & 59 & 12 \\
Estrogens & 42 & 11 \\
Opiates & 42 & 5 \\
Tetracycline & 34 & 2 \\
Cytarabine & 26 & 4 \\
Steroids & 25 & 1 \\
Sulfamethoxazole/trimethoprim & 24 & 1 \\
Sulfasalazine & 23 & 5 \\
Furosemide & 21 & 3 \\
Sulindac & 21 & 8 \\
\hline
\end{tabular}

Table 2. Drugs with a definite causality as reported in several case studies $[2,7,11,13,14]$

\begin{tabular}{lclll}
\hline Drugs & Cases & Definite & Death & Reported time intervals \\
\hline Azathioprine & 19 & 7 & 1 & 10 days to 13 months \\
Cimetidine $^{\text {Codeine }}{ }^{1}$ & 1 & 1 & 0 & 5 days \\
Interferon $\alpha^{\text {Methyldopa }}$ & 6 & 2 & 0 & 1 day to 3 months \\
Metronidazole & 3 & 1 & 0 & 15 weeks \\
Phenylbutazone/oxyphenbutazone & 2 & 2 & 0 & 2 weeks \\
Statins & 1 & 1 & 0 & 1 day \\
Valproate $^{3}$ & 2 & 1 & 0 & 21 days \\
5-ASA & 3 & 1 & 0 & 2 months to 1 year \\
& 6 & 2 & 2 & 1.5-17 years \\
\hline
\end{tabular}

${ }^{1}$ In the 2 cases of definite association reported by Barreto et al. [11], a combination medication of codeine and ibuprofen was used. ${ }^{2}$ Time intervals were only reported in the study by Eland et al. [14]. ${ }^{3}$ Definite association was seen only with simvastatin as reported by Andersen et al. [7]. ${ }^{4}$ Definite association was reported for mesalazine ( 4 cases) and olsalazine (1 case). 


\begin{tabular}{r|l|l|l}
$\begin{array}{r}\text { Case Reports in } \\
\text { Gastroenterology }\end{array}$ & $\begin{array}{l}\text { Case Rep Gastroenterol 2012;6:223-231 } \\
\text { DOI: 10.1159/000338652 }\end{array}$ & $\begin{array}{l}\text { Published online: } \\
\text { May 3, 2012 }\end{array}$ & $\begin{array}{l}\text { @ 2012 S. Karger AG, Basel } \\
\text { ISSN 1662-0631 } \\
\text { www.karger.com/crg }\end{array}$ \\
\hline
\end{tabular}

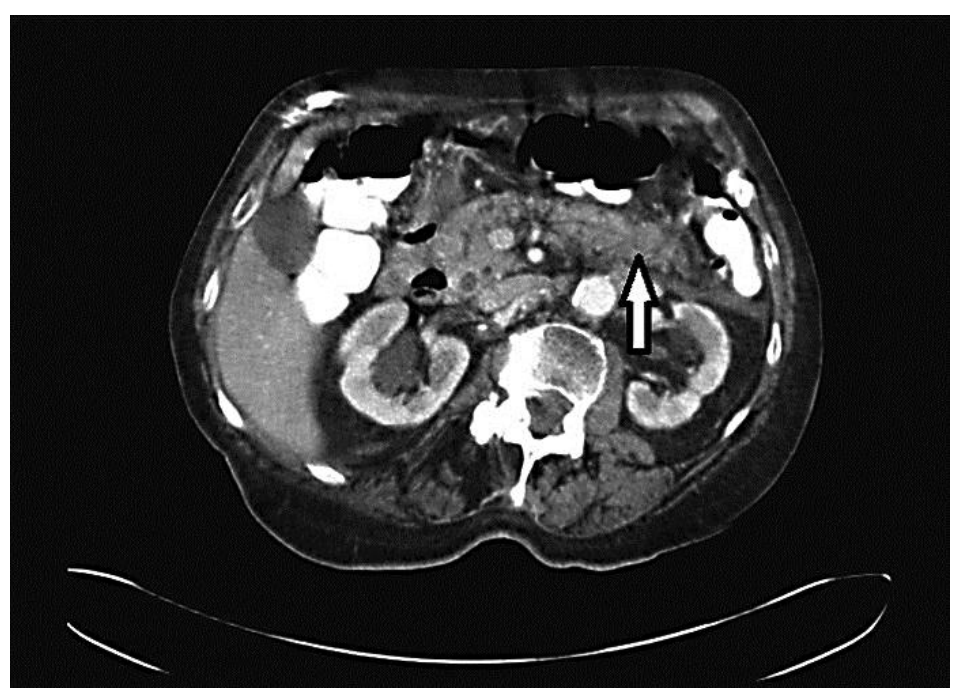

Fig. 1. Computed tomography showing the pancreas (arrow) with trace amounts of surrounding fluid and peripancreatic fat stranding, suggestive of acute pancreatitis. 


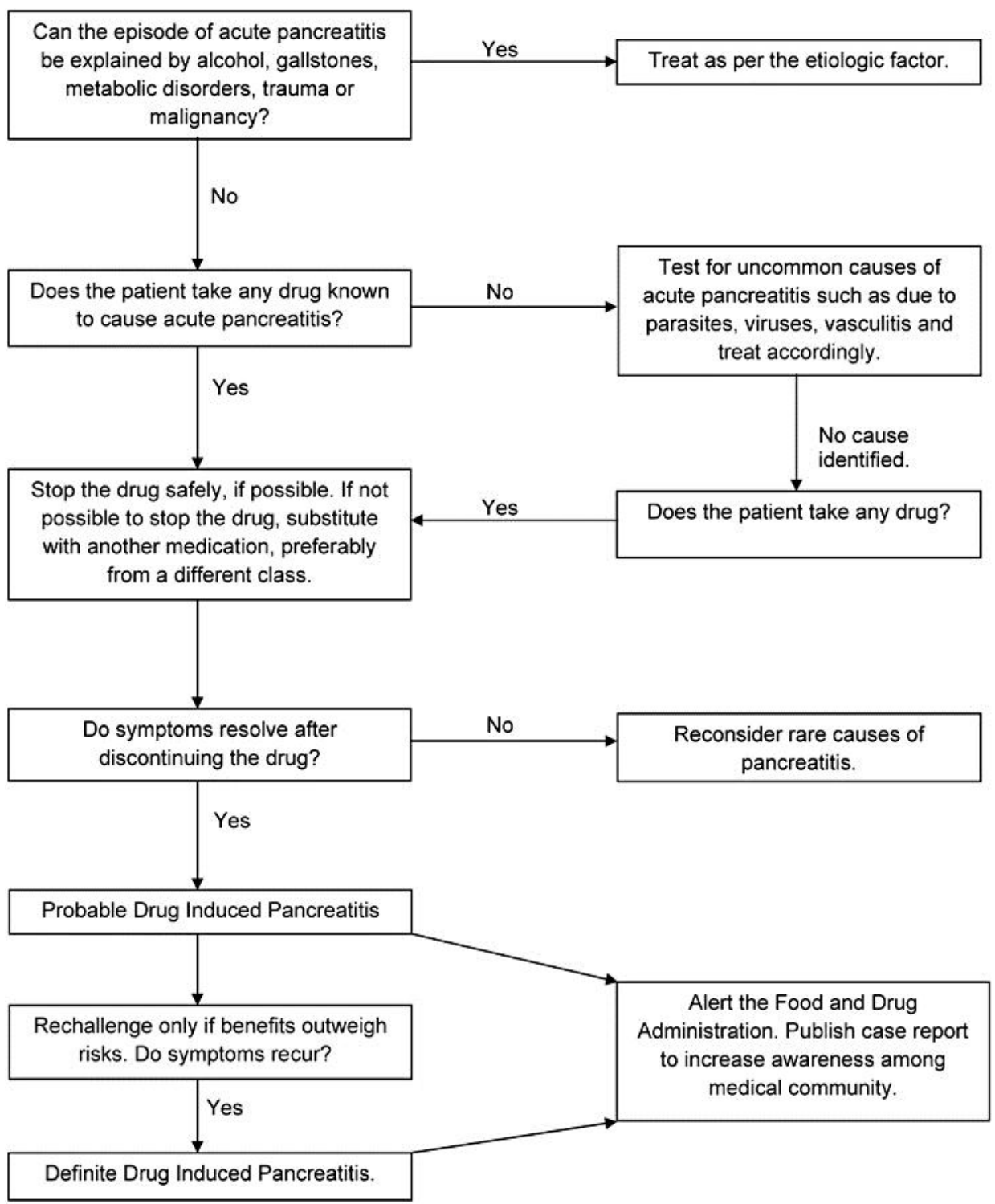

Fig. 2. Algorithm for the management of DIP.

\section{References}

1 Tenner S, Steinberg WM: Acute pancreatitis; in Feldman M, Friedman LS, Brandt LJ (eds): Sleisenger and Fordtran's Gastrointestinal and Liver Disease, ed 9. Philadelphia, Saunders, 2010, pp 959-983.

-2 Vinklerova I, Prochazka M, Prochazka V, Urbanek K: Incidence, severity, and etiology of drug induced acute pancreatitis. Dig Dis Sci 2010;55:2977-2981.

-3 Taguchi M, Yokota M, Koyano H, Endo Y, Ozawa Y: Acute pancreatitis and parotitis induced by methimazole in a patient with Graves' disease. Clin Endocrinol 1999;51:667-670.

4 Su DW, Zou DJ, et al: One case of methimazole induced acute pancreatitis. Chin J Pract Med 2008;24: 2915.

5 Yang M, Qu H, Deng HC: Acute pancreatitis induced by methimazole in a patient with Graves' disease. Thyroid 2012;22:94-96. 
6 Trivedi CD, Pitchumoni CS: Drug-induced pancreatitis: an update. J Clin Gastroenterol 2005;39:709-716.

7 Andersen V, Sonne J, Andersen M: Spontaneous reports on drug-induced pancreatitis in Denmark from 1968 to 1999. Eur J Clin Pharmacol 2001;57:517-521.

-8 Lancashire RJ, Cheng K, Langman MJS: Discrepancies between population based data and adverse reaction reports in assessing drugs as causes of acute pancreatitis. Aliment Pharmacol Ther 2003;17: 887-893.

9 Nitsche CJ, Jamieson N, Lerch MM, Mayerle JV: Drug induced pancreatitis. Best Pract Res Clin Gastroenterol 2010;24:143-155.

10 Norgaard M, Ratanajamit C, Jacobsen J, Skriver MV, Pedersen L, Sorensen HT: Metronidazole and risk of acute pancreatitis: a population based case control study. Aliment Pharmacol Ther 2005;21:415-420.

11 Barreto SG, Tiong L, Williams R: Drug induced acute pancreatitis in a cohort of 328 patients. A single center experience from Australia. J Pancreas 2011;12:581-585.

12 Karch FE, Lasagna L: Adverse drug reactions. A critical review. JAMA 1975;234:1236-1241.

13 Lankisch PG, Droge M, Gottesleben F: Drug induced acute pancreatitis: incidence and severity. Gut 1995;37:565-567.

14 Eland IA, van Puijenbroek EP, Strukenboom MJMC, et al: Drug associated acute pancreatitis: Twenty-one years of spontaneous reporting in the Netherlands. Am J Gastroenterol 1999;94:2417-2422.

15 Spanier BWM, Tuynman HARE, Van der Hulst RWM, et al: Acute pancreatitis and concomitant use of pancreatitis associated drugs. Am J Gastroenterol 2011;106:2183-2188. 